\title{
Sozialmedizin in Zeiten der Corona-Pandemie
}

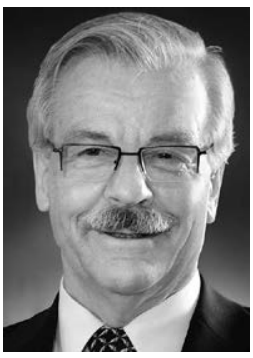

Johannes Gostomzyk

Ehrenpräsident der Deutschen Gesellschaft

für Sozialmedizin und Prävention (DGSMP)
Bibliografie

DOI https://doi.org/10.1055/a-1099-8223

Gesundheitswesen 2020; 82: 379-380

(c) Georg Thieme Verlag KG Stuttgart · New York

ISSN 0941-3790

Korrespondenzadresse

Prof. Dr. Johannes Gostomzyk

Schwangaustraße 2

86163 Augsburg

johannes.gostomzyk@gmx.de
Die gesundheitlichen Auswirkungen und Folgen der CoronavirusPandemie (Covid-19) sind derzeit nicht vorhersagbar. Als Dauer der Pandemie wurde von Seiten des RKI ein Zeitraum von bis zu 2 Jahren geschätzt. Aktuelle Lagebeurteilungen, Prognosen und Bewertungen getroffener Maßnahmen müssen ständig überprüft und angepasst werden und sollten auch nützlich sein.

Einrichtungen mit sozialmedizinischen Aufgaben wie Gesundheitsämter, sozialmedizinische Dienste, einschlägige Beratungsstellen in Kliniken usw. und ihre Mitarbeiter mit sozialmedizinischer Kompetenz, dazu gehören auch die Mitglieder der DGSMP in ihren jeweiligen Tätigkeitsbereichen, leisten wichtige Beiträge zur Krisenbewältigung und sind dabei oft auch Vorbild. Ihre Aufgaben betreffen insbesondere die Wechselwirkungen zwischen der gesundheitlichen Situation der Bevölkerung, der Wirksamkeit des Gesundheitssystems im Verlauf der Pandemie und den pandemiebezogenen Aktivitäten in der Gesellschaft. Bisher fehlt eine kausale Therapie gegen das Corona-Virus.

Zur Förderung der Akzeptanz in der Bevölkerung und der Effektivität notwendiger, das tägliche Leben belastender Einschränkungen und Verbote zur Bewältigung der Coroner-Krise, können sozialmedizinische Orientierung und präventivmedizinisches Wissen und Handeln in folgenden 3 Bereichen wesentliche Beiträge leisten:

1. Information und Kommunikation

2. Versorgungsgerechtigkeit, Versorgungssysteme

3. Solidarität, Gesundheitschancen für alle

\section{Information und Kommunikation}

Sozialmedizin verfügt über wissenschaftliche Methoden und Erfahrungen in der Epidemiologie und der Gesundheitsberichterstattung (GBE) sowie der Transformation der Erkenntnisse auf die jeweilige Handlungsebene.

Menschen brauchen in der Zeit der Pandemie, bestmögliche, umfassende, fachlich fundierte Informationen zu Fakten, Bewer- tungen und Perspektiven aus vertrauenswürdigen Quellen, insbesondere der Wissenschaft, um Maßnahmen der Prävention und präventives Verhalten zu verstehen und ohne äußeren Zwang in ihr Leben zu integrieren, um vermeidbare Ängste abzubauen und Immunität zu entwickeln gegen Fake News und gezielte Fehlinformationen.

In kurzen Abständen neu erhoben Fakten und bevölkerungsbezogene Aussagen zur Pandemie dienen dem Gesundheitsschutz. Insbesondere vorläufige Lagebeurteilungen in einem dynamischen Prozess bedürfen der sorgfältigen fachlichen Interpretation ihrer Aussage, um unbegründete oder gezielte Fehldeutungen zu verhindern.

Gesundheitsbezogene Informationen, Befunde und Bewertungen der Bundesebene bedürfen für die Übertragung ihrer Wertigkeit auf die Landesebene und die kommunale Ebene, der in besonderem Maß bevölkerungsrelevanten Handlungsebene, der sorgfältigen sachverständigen Prüfung und zielgerichteten Anwendung vor Ort. Besonders in der Zeit der Pandemie sind kommunale Institutionen mit sozialmedizinischer Kompetenz wie Gesundheitsamt, Sozialamt, Krankenkassen, Selbsthilfeeinrichtungen und andere lokale Akteure zur Kooperation untereinander und mit den Bürgerinnen und Bürgern aufgerufen, im Interesse der Gesundheit aller.

\section{Versorgungsgerechtigkeit, Versorgungssysteme}

Sozialmedizin verfügt über normatives Wissen aus medizinischer Ethik, aus Medizin- und Sozialrecht und zur Struktur und Funktion des Gesundheitssystems. Angesichts der Corona-Pandemie mit rasanten Veränderungen der öffentlichen Gesundheit und befürchteter Überlastung stationärer Behandlungseinrichtungen gilt es, gesundheitspolitische Entscheidungen der Bevölkerung zu erklären. Das Coronavirus hat die Welt im Griff und schafft für jeden Einzelnen eine vollkommen neue Situation bezüglich der Sorge füreinander und um die eigene Gesundheit. 
Der bisherige Verlauf der Corona-Krise hat erhebliche Defizite in der präventiven Gesundheitsversorgung (Fehlen von Schutzausrüstungen, Desinfektion u. a.) aufgezeigt und sowohl prosoziales als auch egoistisches Verhalten von Menschen als Reaktion darauf offengelegt. Unsoziales Verhalten gefährdet die Umsetzung von Gerechtigkeit in der gesundheitlichen Versorgung, das Recht jedes Menschen auf subsidiäre Solidarität hinsichtlich der bestmöglichen medizinischen Versorgung gegenüber existenzbedrohenden Risiken.

Das Urteil über eine gerechte Verteilung knapper Ressourcen fällt in der Bevölkerung offensichtlich kontrovers aus, wenn es zu Einschränkungen der eigenen Bedürfnisbefriedigung kommt. In Mangelsituationen ist das ethische Problem der Versorgungsgerechtigkeit im Gesundheitswesen im Wesentlichen ein Verteilungsproblem. Eine sozialverträgliche Verteilung von Gesundheitsleistungen erfolgt unter der Maxime der Priorisierung entsprechend der Bedarfsgerechtigkeit abhängig vom Hilfebedarf.

Die Deutsche Interdisziplinäre Vereinigung für Intensiv- und Notfallmedizin hat am 25.032020 klinisch-ethische Empfehlungen zu „Entscheidungen über die Zuteilung von Ressourcen in der Notfall- und der Intensivmedizin im Kontext der COVID-19-Pandemie“ vorgelegt. Dazu wurden auch Verfahren und Kriterien für Priorisierungsentscheidungen formuliert.

Auch in der gesundheitsbezogenen Prävention ist Priorisierung ein Verfahren, in dem die Vorrangigkeit bestimmter Schutzmaßnahmen, z. B. die Expositionsvermeidung gegenüber dem Coronavirus, vor anderen Interessen festzustellen ist. Die Akzeptanz der Priorisierung in der Prävention und in der Gesundheitsversorgung hängt, neben der Transparenz im Vorgehen, maßgeblich davon ab, wer priorisiert und mit welcher Legitimation und nach welchen Kriterien dies in der konkreten Situation, z. B. bei einem Mangel an Beatmungsplätzen geschieht.

\section{Solidarität, Gesundheitschancen für alle}

Solidarität mit den Bedürftigen ist die Begründung für die Forderung nach einer sozialen Medizin. Während der Corona-Pandemie erfährt der Begriff „Solidarität“ in der öffentlichen Diskussion eine Renaissance. Solidarisches Handeln erhält eine neue Wertigkeit in der einschließlich ihrer Zukunftsperspektive verunsicherten Gesellschaft.

Solidarität im Gesundheitsbereich steht primär als Leitmotiv für Verbundenheit und Hilfsbereitschaft des für seine Aufgaben hochmotivierten Personals der Gesundheits- und Pflegedienste mit hilfesuchenden Menschen. Bei vielen Mitarbeitern im Gesundheitswesen (Pflegende, Ärzte und Angehörige anderer Heilberufe) initiiert aber der Begriff Solidarität auch eine ethisch-politische Begründung für ein neues Zusammengehörigkeitsgefühl der Betroffenen nach einer für sie immer belastender werdenden Epoche der Ökonomisierung des Systems mit neoliberal geprägten Rentabilitätsvorgaben.

Solidarität in der Gesellschaft zum Schutz der Gesundheit umfasst auch den Arbeitsschutz für die Mitarbeiter im Gesundheitswesen. Das umfasst einen ausreichenden Gesundheitsschutz gegenüber den Gefahren durch das Corona-Virus SARS-CoV-2 und ebenso einen wirksamen Schutz vor einem Burnout der Mitarbeiter in Beratungs- und Pflegediensten durch übermäßige Belastungen, insbesondere bei chronischem Personalmangel.

Die Diskussion über die Bewältigung der Corona-Pandemie in der Bevölkerung und unter den Mitarbeitern des hochgradig belasteten Gesundheitswesens beinhaltet aber auch die Chance zur Festigung der Solidarität in der Gesellschaft in der Zukunft. - Ohne dass wir genau wissen, wann diese beginnt: die Grundlagen dafür werden schon heute gelegt.

\section{Interessenkonflikt}

Die Autorinnen/Autoren geben an, dass kein Interessenkonflikt besteht. 